\title{
Revisando críticamente el discurso sobre el impacto de la sociedad de la información en la población infantil: el problema del aislamiento social
}

\section{Iván Rodríguez Pascual}

Universidad de Huelva. Departamento de Sociología y Trabajo Social

Campus del Carmen. Av. Fuerzas Armadas, s/n. 21071 Huelva

ivan@uhu.es

\section{Resumen}

Las nuevas tecnologías de la información y la comunicación (TIC) constituyen la evidencia de un conjunto de transformaciones que están teniendo lugar en la vida cotidiana de las personas, que agrupamos bajo el concepto de sociedad de la información. Sin embargo, el análisis de esta nueva sociedad de la información sólo está considerando superficialmente su impacto en ciertas áreas, como es el caso de su penetración en los hogares y el impacto sobre la vida social de los menores. Y el diagnóstico ofrecido por la literatura especializada resulta fuertemente mediatizado por una representación social del menor que podría contribuir a sobredimensionar el riesgo de las nuevas tecnologías, especialmente en lo que se refiere a la posibilidad de que éstas estén contribuyendo a aislar a los menores. En este texto mostramos los hallazgos de un estudio exploratorio de naturaleza cualitativa en el cual participaron niños de entre 6 y 13 años, en el que constatamos la divergencia entre la realidad que aparece ante el investigador y la descripción alarmista que rodea a la sociedad de la información.

Palabras clave: sociedad de la información, tecnología, infancia.

\section{Abstract. A Critical Approach to the Speech about Children and Information Society: the Problem of Social Isolation}

New technologies of information and communication (TICs) are the evidence of a wide range of changes that are taking place in the everyday life of people that we describe with the name information society. However the analysis of this new information society is considering only superficially the impact of TICs in certain domains, as it is the case of households and social life of children, and the speech about the impact of this information society is affected by the social representation of childhood when social isolation is noticed. In this text we show the findings that resulted of an exploratory qualitative study in which children from 6 to 13 years old took part to explain us how these new technologies had affected their lives, finding a sensible divergence between reality and diagnosis.

Key words: information society, technology, childhood. 


\section{Sumario}

1. La sociedad de la información: conceptos borrosos para fenómenos incipientes

2. El impacto de las TIC y la sociedad de la información en las vidas de los niños: un diagnóstico
3. Los menores hablan sobre la tecnología

4. A modo de conclusión

Bibliografía

\section{La sociedad de la información: conceptos borrosos para fenómenos incipientes}

Usamos el término sociedad de la información para describir la realidad incipiente y cambiante que nos deja el viejo siglo XX, preferentemente en un contexto europeo. Una realidad en la que las nuevas tecnologías de la información y la comunicación (en adelante, TIC) desempeñan un papel central. Sin embargo, la realidad no se corresponde por entero con los múltiples conceptos (sociedad de la información, sociedad digital, mesmerizada, etc.) que utilizamos para retratarla. En parte debido a que, precisamente por su carácter emergente, las predicciones tienden a confundirse con los diagnósticos, y ambas se sustentan con demasiada frecuencia sobre la mixtificación de muchos de sus rasgos y el trabajo con conjeturas de alcance ciertamente inverificable (por ahora). Este es un problema especialmente acuciante desde el mismo momento en que el término ha sido aceptado y difundido en el ámbito político, a pesar de sus fisuras teóricas (Bericat 1996; Kofler 1998).

Por todo ello nos parece interesante contar con definiciones cercanas y operativas, que permitan la investigación sobre el terreno, máxime cuando trabajamos con un colectivo particular que demanda una aproximación metodológica también particular, como es el caso de la población infantil. A la hora de plantear el estudio cuyos resultados aquí presentamos consideramos, siguiendo los criterios del propio Instituto Nacional de Estadística (2001: 7), que la sociedad de la información es aquella que hace un uso generalizado de las redes de información que proporcionan grandes cantidades de datos, de bienes, servicios de la información y de la comunicación y contenidos de la información. Teniendo en cuenta que las nuevas tecnologías de la información y la comunicación están en el centro de este proceso de transformación, su implantación y uso se convierte en un indicador muy significativo de la aproximación de una sociedad cualesquiera al modelo de sociedad de la información, considerando también que la población infantil no ha permanecido ajena a la difusión de las nuevas tecnologías de la información y la comunicación, sino todo lo contrario, como sostendremos más adelante basándonos en los datos de su implantación en la sociedad española. Por supuesto, la imprecisión del propio concepto «infancia», que no se corresponde exactamente con el de «menores de edad", agudiza los problemas operativos, a lo que hay que añadir que la 
mayor parte de las fuentes consultadas carecen de un criterio consistente a la hora de delimitar tal grupo de edad.

En definitiva, el objetivo de este trabajo es el de explorar un aspecto poco tratado del proceso general de transformación hacia la sociedad de la información: el impacto que éste genera en la población infantil y sus modos de vida, y muy especialmente sobre las redes de sociabilidad que constituyen el marco característico de la vida infantil. Hemos partido de la hipótesis de que el discurso general sobre la llegada de la sociedad de la información y su impacto sobre la vida cotidiana de los menores tiende a recalcar las amenazas que ésta representa, sobredimensionando su impacto perjudicial en el contexto de la infancia, debido principalmente a que la representación social del menor en nuestra sociedad lo identifica como un agente extremadamente vulnerable, indefenso y fácilmente manipulable. Proponemos que el análisis del discurso infantil —el que los propios menores producen en un marco de libre intervención como el de la entrevista grupal- debe contribuir a clarificar hasta qué punto esta representación que se encuentra tras el diagnóstico del impacto de las TIC sobre la población infantil responde a la realidad o ha magnificado ciertos aspectos de nuestra existencia como niños y ocultado otros igualmente relevantes. Nos hemos centrado en la cuestión de si las nuevas tecnologías son las causantes de aislamiento social y empobrecimiento de la sociabilidad entre los niños, aunque otros muchos aspectos parciales del problema podrían haber servido para efectuar el mismo contraste.

\section{El impacto de las TIC y la sociedad de la información en las vidas de los niños: un diagnóstico}

\subsection{La cuestión del acceso: ¿son los niños usuarios de las TIC?}

La primera pregunta interesante que podemos plantearnos es si los niños participan activamente de eso que venimos denominando la sociedad de la información, y que en términos operativos hemos identificado con la difusión de las TIC tanto en el ámbito productivo como en los hogares. A este respecto, y en líneas generales, podemos considerar que la tendencia en la sociedad española es hacia el crecimiento de la penetración de estas nuevas tecnologías.

Tal y como señalan diversas fuentes, entre ellas el conocido Estudio General de Medios ${ }^{1}$ que elabora la Asociación para la Investigación de los Medios de Comunicación (AIMC), algunas de las principales aplicaciones de las TIC tienen ya una presencia significativa y creciente en el contexto de la sociedad española. Así, constatamos que una cuarta parte de los hogares españoles tienen acceso a la red, y casi la misma proporción de españoles son usuarios de la misma. La proporción de usuarios de la red sobre la población total creció 
desde un modesto 2,8\% en 1997 a más de un 33\% en 2004, y lo mismo puede decirse de la proporción de usuarios de Internet que se conectan desde su propio hogar, en detrimento de su centro de trabajo o estudios: de un $36,4 \%$ al $62 \%$ en ese mismo período. Pero, ¿cuál es la magnitud del fenómeno entre los niños y niñas españoles? Como ya hemos indicado, distintas fuentes consideran distintos intervalos de edad, lo que dificulta la obtención de datos consistentes, aunque no imposibilita que obtengamos una imagen significativa de la extensión de las TIC entre la población infantil. El mismo Estudio general de medios señala cómo el número de usuarios de entre 14 y 19 años se sitúa alrededor de un $16 \%$ sobre el conjunto de usuarios de todas las edades, casi el doble que en $1997 . .^{2}$ Éste es un dato general que oculta indicios más relevantes conforme se amplia el abanico de los distintos productos tecnológicos analizados (incluyendo, por ejemplo, la telefonía móvil) y se reduce la edad de los sujetos entrevistados. La propia AIMC (2004) daba a conocer recientemente el estudio de Audiencia infaltil/juvenil de medios, ${ }^{3}$ basado en más de 4.000 entrevistas personales, según el cual aproximadamente la mitad de los niños entre 8 y 13 años navegan con frecuencia por Internet y un tercio de los mismos dispone de teléfono móvil para su uso personal. Según dicho estudio, cada año en que incrementamos la edad del usuario es más probable que esté en contacto con estos nuevos productos tecnológicos y que haya crecido el equipamiento de los hogares que habitan. Una visión más completa se encuentra contenida en la Encuesta sobre tecnologías de la información en los hogares, a cargo del Instituto Nacional de Estadística (2003), y en la que el intervalo de edades considerado se encuentra entre los 10 y los 14 años.

Los datos son suficientemente elocuentes: el grado de penetración de las nuevas tecnologías de la información y la comunicación en la vida de los niños es ya notable. El uso de ordenador e Internet ya no es extraño para una inmensa mayoría de menores entre 10 y 14 años, al tiempo que otro tipo de aplicaciones de las nuevas tecnologías se encuentran muy difundidas entre la población infantil, como es el caso de la telefonía móvil (tabla 1). De otro lado, la cuantificación de este nuevo fenómeno viene a echar por tierra la idea de que estas tecnologías tienen un uso predominantemente lúdico. Tal y como se aprecia en la tabla 2, los niños reparten su tiempo frente al ordenador o Internet alternando las actividades de ocio con aquéllas propiamente académicas, aunque en el caso de los varones la diferencia parece resolverse más acusadamente a favor del ocio.

De la misma manera, nos parece que la rapidez con que determinados equipamientos tecnológicos se han popularizado entre los menores no es ajena a

2. La propia AIMC sugiere que al comparar este dato con el de la audiencia infantil de Internet obtenemos una tasa de penetración entre la población menor de 14 años equivalente a un $48,9 \%$, mientras que la de la población de 14 o más años apenas supera el 33\%. Esto significa que, en términos relativos, la población infantil cuenta con un peso determinante en la composición del conjunto de los usuarios de la red.

3. Disponible también on-line en el sitio www.aimc.es 
Tabla 1. Acceso de los niños españoles de 10 a 14 años a las nuevas tecnologías (\%).

\begin{tabular}{llll}
\hline & Niños & Niñas & Total \\
\hline Usuarios de ordenador en los últimos 3 meses & 58,3 & 60,3 & 59,3 \\
Usuarios de Internet en los últimos 3 meses & 49,16 & 51,9 & 50,4 \\
Niños que disponen de teléfono móvil & 51,9 & 40,2 & 34,3 \\
\hline
\end{tabular}

Fuente: INE (2003).

Tabla 2. Uso de las TIC entre los niños españoles de 10 a 14 años (\%).

\begin{tabular}{llll}
\hline & Niños & Niñas & Total \\
\hline Utilización del ordenador para ocio & 93,6 & 90,3 & 92 \\
Utilización del ordenador para trabajos escolares & 84,2 & 85,5 & 84,8 \\
Otros usos del ordenador & 12,5 & 11,1 & 11,8 \\
Utilización de Internet para ocio & 75,3 & 69,9 & 72,6 \\
Utilización de Internet para trabajos escolares & 77,4 & 80,2 & 78,8 \\
Otros usos de Internet & 16 & 14,7 & 15,4 \\
\hline
\end{tabular}

Fuente: INE (2003).

la peculiar configuración del espacio social en el que éstos se mueven habitualmente. Hablamos de niños que pasan gran parte de su tiempo confinados en algún tipo de ambiente institucional, preferentemente el escolar, que han visto su tiempo de ocio fuertemente compartimentalizado por actividades extraescolares, deberes para casa, horarios de trabajo de sus progenitores, etc. y que al mismo tiempo tienen restringido el acceso al juego libre fuera de las fronteras domésticas. En estas circunstancias no sorprende tanto la proliferación de videoconsolas y videojuegos para PC, más todavía en los hogares españoles, donde la peculiaridad de una sociedad de familias nucleares que ha postergado la natalidad deja a los menores con pocas posibilidades de interacción con hermanos y otras figuras adultas distintas de sus padres (Juárez, 1998).

Al hilo de esta nueva realidad que afecta a la población infantil, ha surgido todo un discurso que alerta de los riesgos y consecuencias de la llegada de la sociedad de la información, del que hemos partido a la hora de contrastar el diagnóstico presente en la literatura especializada con la realidad tal y como es retratada por nuestros informantes. En los restantes apartados de este trabajo procedemos a caracterizar tal discurso, para posteriormente analizar la información generada por el trabajo de campo y las posibles divergencias entre ambos.

\subsection{El diagnóstico sobre el impacto del uso de las TIC en los niños}

La irrupción de Internet y otros desarrollos tecnológicos asociados ha avivado considerablemente el debate en torno a las condiciones de vida de la infancia 
en las sociedades modernas, y lo ha hecho precisamente en lo que se refiere a la posibilidad de que las actividades de los menores en el ciberespacio estén escapando al control de los adultos. ${ }^{4}$ Es lógico, en este contexto, que hayan proliferado igualmente las iniciativas de investigación relacionadas con el problema del impacto de estas nuevas tecnologías sobre la vida de los menores, aunque éstas han sido planteadas desde perspectivas diversas y con objetivos muy diferentes.

En el terreno de la investigación sobre las consecuencias de la implantación de las TIC la tónica no ha sido diferente. Durante mucho tiempo hemos contado con modelos extremadamente reduccionistas que buscaban representar la relación entre el niño y la tecnología, lo que finalmente ha sido conocido (y criticado) bajo la denominación HCI (Human Computer Interaction), donde la complejidad cultural de la vida infantil ha sido sustituida por diseños experimentales o estudios parciales que, desde una perspectiva sociológica, se nos antojan claramente insuficientes. ${ }^{5}$ Recientemente, nuevos enfoques que se alejan un tanto de este modelo HCI han aparecido en escena $y$, cada vez en mayor medida, encontramos investigaciones que buscan estudiar al niño en su propio contexto cultural evitando en lo posible la adopción de un punto de vista adultocéntrico y/o experimental (Jessen 1999; Yates y Littleton 1999; Holloway y Valentine 2001).

A la hora de destacar el diagnóstico que emana de la literatura científica en relación a las posibles consecuencias de la implantación de las TIC en el contexto de la vida infantil no podemos pasar por alto la naturaleza imprecisa y hasta contradictoria del mismo. Así, no es extraño encontrar un discurso contradictorio en el que, por un lado, se alaban las virtudes del progreso tecnológico y se insta a la población para que se alfabetice en el uso de las nuevas tecnologías mientras que, por otro, se alerta sobre el peligro que éstas suponen para los niños, presentándose a éstos como a usuarios inmaduros que deben ser protegidos a toda costa apartándolos de la nueva sociedad red (Fager et al. 2001). La profusión de elementos tecnológicos, la invasión de los medios en los hogares, ha significado, para algunos, el fin de la propia infancia (Postman 1984). Igualmente, no ha faltado quien ha señalado que los nuevos hábitos de la población infantil en lo que respecta al uso de estas nuevas tecnologías son francamente perjudiciales, puesto que producen una amplia gama de conse-

4. De hecho, algunos autores han sugerido que la denuncia de estas amenazas potenciales de las nuevas tecnologías de la información y la comunicación es una repetición del discurso generado décadas atrás al producirse otro tipo de innovaciones tecnológicas como la televisión o el cine, con lo que nos encontraríamos ante una situación novedosa pero con los mismos viejos temores referidos a la «contaminación» de la infancia a través de contenidos supuestamente peligrosos, tal y como queda reflejado en Wartella y Jennings (2000).

5. El estudio de los grupos de pares y su papel en el marco de la cultura infantil constituye unos de los pilares del acercamiento sociológico a la infancia, pero desborda los reducidos límites de este artículo. No obstante, respecto a esta cuestión pueden consultarse algunas referencias fundamentales, como las de Jessen (1999), Holloway y Valentine (2001), Montgomery (2000) y Orleáns y Laney (2000). 
cuencias entre las que cuentan la obesidad, adicción, ansiedad, depresión, deshumanización de las relaciones sociales y, por supuesto, aislamiento social (Moll 1998; Nissenbaum y Walker 1998; Subrahmanyan et al. 2000). La trascendencia de estos datos ha quedado oscurecida por uno de los grandes debates que han acompañado a la difusión del uso de Internet: la cuestión del acceso a contenidos de riesgo (especialmente la pornografía), que viene generando un volumen muy significativo de referencias de las que aquí sólo ofrecemos una pequeña muestra (Freeman-Longo 2000; Hicks y Halping 2001; Mc Cabe 2000; Oswell 1998; Stanley 2001; Tien 1994).

El estudio del impacto de las TIC y la nueva sociedad de la información sobre la población infantil y sus condiciones de vida se mueve, por tanto, en circunstancias ambiguas, dado que se abre un campo nuevo que presenta diversas amenazas potenciales para la infancia sin que éstas hayan sido suficientemente tamizadas empíricamente al acudir a los propios niños como informantes y/o unidades de observación, al menos no desde una perspectiva participativa que no anule su discurso o no busque su objetivación desde un criterio adulto. En nuestro caso, nos hemos centrado en la cuestión del impacto de las TIC sobre la vida social de los niños, comparando el diagnóstico que ofrece la literatura especializada que vincula las TIC con el problema del aislamiento social y la ruptura de las cadenas de sociabilidad en la infancia, con el discurso producido por los propios niños en condiciones no experimentales. A continuación ofrecemos los detalles metodológicos y los principales hallazgos del estudio.

\section{Los menores hablan sobre la tecnología}

\subsection{Notas metodológicas}

Presentamos los resultados de un estudio de carácter cualitativo que pretendía indagar en las pautas de uso y disfrute de la tecnología por parte de los menores, cuyo propósito era el de recoger sobre el terreno el discurso que los mismos menores producen en torno a esta cuestión para compararlo con el producido en la literatura especializada.

Para tal fin se realizaron en la ciudad de Huelva ${ }^{6}$, en diferentes momentos temporales a lo largo del año 2001 y 2002, un total de siete entrevistas grupales con niños y niñas cuyas edades oscilaban entre los 6 y los 14 años, constituidos en grupos de entre 6 y 10 sujetos a los que el entrevistador proponía

6. Huelva constituye un ejemplo bastante claro del tipo de crecimiento urbano generado en la segunda mitad del siglo XX en España. Apenas tiene 140.000 habitantes, procedentes en su mayor parte de los fuertes procesos migratorios que tienen lugar en el marco de la geografía española durante los años sesenta. Actualmente la ciudad ha experimentado un fuerte proceso de modernización y reconversión desde una actividad primordialmente industrial a otra de sector servicios, a lo que ha servido la creación en el año 1993 de la universidad y su posicionamiento como centro administrativo. En general, el nivel de implantación de las TIC en la ciudad es superior al de la media andaluza y se encuentra ligeramente por debajo de la media española. 
Tabla 3. Esquema de diseño de los grupos de discusión.

\begin{tabular}{llll}
\hline Edad & Hábitat & \\
\cline { 2 - 4 } $\begin{array}{c}\text { Niños } \\
(6-11)\end{array}$ & Rural & Semiurbano & Urbano \\
\hline $\begin{array}{c}\text { Prupo } \\
\text { Preadolescentes } \\
(12-14)\end{array}$ & $\begin{array}{c}1 \text { grupo } \\
\text { (majo) } \\
1 \text { grupo }\end{array}$ & $\begin{array}{c}1 \text { grupo } \\
\text { (medio) }\end{array}$ \\
\hline
\end{tabular}

Fuente: elaboración propia.

hablar sobre su tiempo libre en general, derivando poco a poco la conversación hacia la cuestión del equipamiento tecnológico de sus hogares y la forma de uso del mismo. ${ }^{7}$ El diseño de estos grupos perseguía el poder diferenciar entre el discurso de los chicos que habitaban un entorno plenamente urbano y aquellos que situábamos en un medio rural o semiurbano, ${ }^{8}$ de la misma manera que también distinguimos entre preadolescentes (mayores de 12 años) y niños (menores de 12), a lo que añadimos el criterio del origen social de los menores tal y como muestra la tabla 3:

La elección de los grupos vino determinado por varios criterios básicos: por un lado buscamos realizar las entrevistas con grupos preexistentes (por ejemplo, grupos que se reunían para realizar actividades extraescolares en asociaciones de vecinos o centros sociales), por otro que la composición de éstos fuera un reflejo de los diferentes orígenes sociales de los niños entrevistados. A resultas de esta estrategia contamos con diferentes grupos que se distinguían no sólo por su hábitat y edad, sino por la extracción social de sus participantes. El grupo realizado con niños de hábitat rural y uno de los que tuvieron

7. El estudio de las peculiaridades metodológicas de la investigación con niños es suficientemente interesante para dedicarles un espacio propio, del cual no disponemos en este trabajo. Baste decir que elegimos la entrevista grupal por ser un esquema más abierto y flexible que el de un grupo de discusión o una entrevista en profundidad, pues permitía interactuar a los menores con el investigador y entre ellos simultáneamente. En general, las entrevistas de grupo fueron más espontáneas y difíciles de controlar cuanto más niños de corta edad entraban en su composición, aunque el resultado final fue de gran riqueza discursiva, pese a que muchos de estos niños apenas alcanzaban los diez años. Éstas fueron realizadas en espacios educativos (nunca aulas o laboratorios), centros sociales o en locales de asociaciones de vecinos de la ciudad de Huelva, y en la mayor parte de los casos no hubo más presencia de adultos que la del propio investigador y su ayudante. Sobre la peculiaridad metodológica del trabajo con menores aconsejamos acudir a Fine y Sandstrom (1988); Cook-Gunperz et al. (1986); Graue y Walsh (1998); Greig y Taylor (1999); Holmes (1998); Honig et al. (1999).

8. Que incluía a chicos de la ciudad pero integrados en sus barrios periféricos o suburbiales. 
lugar en entorno semiurbano, en el que contamos con hijos de usuarios de los servicios sociales de la zona, estuvieron compuestos por informantes de origen social bajo o medio-bajo. En el ámbito urbano, por su parte, contamos con un grupo de niños menores de 12 años con origen social medio, cuyos participantes fueron captados en un colegio de titularidad privada. Finalmente se formó, a través del método de la «bola de nieve», un grupo de preadolescentes de hábitat semiurbano con origen social medio-alto y un último grupo de preadolescentes urbanos en el que se experimentó dotándolo de una composición mixta, en el que incluimos dos miembros de origen social medio-alto $y$ tres de origen social bajo. Tal diversidad en el origen social de los participantes, unido a los criterios de zonificación y distribución por edades que antes hemos expuesto, dio lugar a una información rica en matices, si bien dotada de singularidades.

Por ejemplo, fue un rasgo común a todos ellos la producción de un discurso más fragmentado y menos fluido que si el grupo hubiera estado constituido por adultos. ${ }^{9}$ De otro lado, la relación con los menores participantes se vio afectada en ocasiones por interferencias, provocadas por el deseo de los niños de ubicar al investigador como adulto asignándole una posición asumible y explicable por ellos mismos. Por ejemplo, hubo que convencer a los niños antes de comenzar cada grupo que los adultos que allí se encontraban eran ajenos al sistema educativo y que no representaban una amenaza de evaluación sobre lo que decían. ${ }^{10}$

La información producida en el contexto de estos grupos, no obstante, fue extraordinariamente valiosa, y su mayor fragmentación no constituyó un obstáculo cara al análisis en la medida en que estos fragmentos eran generalmente ricos en significado y rápidamente llevaban a otras porciones de sentido que permitían que el análisis ganara en fluidez y matices. A continuación ofrecemos algunas de las evidencias halladas trabajando con estos menores, en concreto aquellas que explican aspectos relevantes que conciernen al estudio del impacto que las nuevas tecnologías de la información tienen sobre las redes sociales en los que se mueve la vida cotidiana de los niños. Para tal fin utilizamos un sistema de notación específico que consiste en asignar una letra para el discurso de los chicos (B) y otra para las chicas $(G)$, designando al investigador con la letra I, junto a la que especificamos la edad del niño y el fragmento de su discurso.

9. El análisis del discurso infantil se realizó contando con apoyo informático, concretamente con el software Atlas.ti

10. Uno de los efectos del internamiento de la población infantil en la escuela, debido a que ésta constituye un ambiente extraordinariamente consistente y permanente en sus vidas, es que éstos interiorizan rápidamente el sistema de recompensas que se les propone y se acostumbran a ser continuamente evaluados por los adultos e incluso por sus propios compañeros, lo que explica este tipo de conductas que describimos. Sobre estos aspectos siempre merece la pena visitar el texto, ya clásico, de Phillip Jackson (1991). 


\subsection{El niño como usuario: del dominio de los medios a la transgresión del dominio adulto}

No querríamos empezar a analizar el discurso infantil sobre las nuevas tecnologías sin hacer referencia a la imagen proyectada por los niños durante las entrevistas de grupo, toda vez que viene a divergir notoriamente con la que la sociedad adulta ha construido y en la que el menor figura como un usuario limitado, ingenuo y extremadamente vulnerable.

Lo que hemos encontrado en el desarrollo de las entrevistas realizadas con los niños es un menor que se ha convertido en usuario activo de la tecnología y que, incluso en el supuesto de no tener acceso a ella en su propio hogar, conoce y es capaz de describir las últimas aplicaciones de las tecnologías de la información y la comunicación. La inmensa mayoría de los niños y niñas entrevistados conocen y han usado alguna vez estas tecnologías y son diestros incluso en el ejercicio de la transgresión de las normas que los adultos imponen a su relación con estos productos tecnológicos, como ellos mismos revelaron. La información recogida viene a avalar esta imagen del niño como usuario activo y cualificado. En los grupos de niños más pequeños (desde los seis años) fueron identificados sin problemas toda una serie de artefactos que el investigador presentaba en forma de fichas (ordenadores fijos y portátiles, escáneres, impresoras, videoconsolas, ratones, webcams, etc.), y allá donde el discurso comienza a enriquecerse y tornarse más fluido encontramos ricas descripciones de estas nuevas tecnologías y sus usos, descripciones que muy probablemente diferirían poco de las que pudiera proporcionar un usuario adulto:

G(13): Es un medio de transmitir información, ¿̨no?

B(11): Para mí Internet es como un libro donde cada página web es un capítulo.

G(13): Son páginas, cada una de un tipo distinto y te metes en la dirección y te enseñan lo que hay metido en ella. Depende de qué página: hay buscadores, hay para divertirse, hay juegos, o información, vamos.

G(13): Pienso que Internet no es una pérdida de tiempo, creo que es algo que nos ayuda a relacionarnos mejor y a hacer más amigos. También nos ayuda a tener más información sobre algo. Creo que Internet es un sitio donde se conoce a mucha gente y puedes hablar con personas de muchos sitios. Lo malo que tiene es que «engancha». Creo que en cada colegio debería haber un ordenador por persona, sería mucho mejor y facilitaría muchas cosas a los niños y a los profesores.

La descripción que los chicos hacen de estos nuevos productos tecnológicos no sólo es profusa, sino que incluye aspectos que los adultos habitualmente consideramos «marginales» o «desviados», y que contemplamos normalmente como un mal uso de la tecnología o un desarrollo particular que conviene desgajar de la misma, mientras que para muchos de los menores con los que hablamos son, en realidad, parte constituyente del nuevo mundo virtual y quizás incluso uno de sus principales atractivos. Y esto vale tanto para hacer nuevos amigos o mantener 
contactos fuera de la influencia y el control paternos como para otros usos ciertamente más radicales, tal y como reflejan las siguientes palabras que aparecieron en uno de los grupos realizados con niños de hábitat urbano y clase media, por regla general usuarios más cualificados y mejor equipados:

I: Básicamente, entonces, [Internet] es un sitio para buscar información, para entretenerse...

G(13): Conocer gente...

$\mathrm{G}(13)$ : ... o fabricar bombas. (Risas.)

Los chicos nos contaron muchas cosas sobre el tipo de uso que dan al PC y a Internet, sobre cómo aprendieron aquello que saben sobre estas nuevas tecnologías de la información y la comunicación, ${ }^{11}$ sobre los beneficios de utilizar la red de redes y también sobre sus peligros, y éstas entre tantas otras cosas. Pero todas ellas han servido para ir apuntalando esa primera impresión que nació con el desarrollo de los primeros grupos: los niños observados en contextos grupales sólo se parecen lejanamente a la representación social del niño como un usuario inexperto y vulnerable, y muestran niveles de destreza que suelen crecer conforme aumenta la edad (preadolescentes) y la extracción social de los mismos (hábitat urbano, origen social medio-alto).

Seguidamente exploramos uno de los aspectos que están relacionados con el impacto de las nuevas tecnologías de la información y la comunicación en la vida infantil. Lo hemos escogido porque aparece recurrentemente en la literatura especializada y constituye una de las posibles amenazas de la extensión de estas tecnologías: conducir a los menores hacia situaciones de aislamiento y pérdida del contacto social como consecuencia del uso compulsivo de las mismas.

\subsection{Impacto sobre las redes sociales: ¿Aislamiento o expansión de una sociabilidad restringida?}

Es una cuestión ampliamente debatida el posible efecto adverso de la tecnología al provocar aislamiento en los menores. La literatura consultada previamente a la realización de las entrevistas grupales con niños bosquejaba una imagen francamente desagradable del usuario: niños que usan compulsivamente su consola de videojuegos y renuncian al ocio compartido; adictos a Internet y frecuentadores de páginas web con contenidos calificados como peligrosos, pero que las más de las veces se identifican con la pornografía; chicos y chicas que han reemplazado el contacto social cara a cara por el pobre

11. Y que normalmente no provenía ni de los padres, que figuran la mayor parte de las veces como figura de apoyo para ocasiones excepcionales (ordenadores que se «cuelgan» o averían, cortes en la conexión, instalación de programas, etc.), ni del curriculum escolar, habitualmente bastante pobre en lo que se refiere a la enseñanza del uso de la tecnología. 
sustituto de una conversación virtual en un chat o a través de la mensajería instantánea. Usuarios atomizados, en fin, que participan del gran engaño de una comunidad virtual que les aparta de las redes sociales a las que deberían pertenecer.

Sin descartar que exista este tipo de sujeto entre la población infantil, las evidencias halladas durante el desarrollo de las entrevistas grupales sugieren una hipótesis explicativa bien diferente. Frente a la interpretación usual de que las TIC generan aislamiento en la población infantil, limitando el contacto social en los niños, proponemos una alternativa: aquella que parte más bien de interrogarse si el aislamiento creciente de los menores y el empobrecimiento de su vida social no son fenómenos preexistentes a las nuevas tecnologías de la información y la comunicación. De esta manera, en lugar de pensar que las TIC producen aislamiento, quizás sea más correcto entender que la razón de que las TIC se hayan introducido tan rápidamente en la vida de los niños es que éstos han visto sus movimientos extremadamente restringidos y han sido ubicados, por tanto, en una situación difícil para producir ocio compartido, por lo que el ordenador o la consola de videojuegos han venido a llenar un hueco significativo en la vida cotidiana de estos niños urbanos que han visto como la calle y el barrio dejaban de ser los espacios privilegiados del juego y el aprendizaje de la vida social.

El discurso infantil gira insistentemente en torno a este problema. A veces revela aspectos de la realidad social en la que se mueven los menores que muy difícilmente entran a formar parte del discurso oficial sobre el impacto de las TIC sobre la vida infantil, generalmente referidos a la manera en que la conducta de los adultos contribuye a construir usos específicos para estas nuevas tecnologías. En este punto se produce una fuerte divergencia entre el diagnóstico adulto de la situación de los menores y el retrato que éstos hacen de su vida cotidiana. Esto, por otra parte, no es nuevo, puesto que viene a ser una constante del mundo desarrollado el construir un concepto de infancia que subestima la autonomía y agencia social del niño y conduce, con demasiada frecuencia, hacia un discurso que demanda una cierta sentimentalización y sobreprotección (Corsaro 1997; Dencik 1992; Jenks 1996; Zelizer 1985). En el contexto de la sociedad española esto ha significado incluso que muchas de las aplicaciones de las nuevas tecnologías de la información y la comunicación se han popularizado entre los menores precisamente por servir a los intereses de los adultos. Es el caso de la telefonía móvil, que constituye hoy un servicio utilizado por gran cantidad de menores. De acuerdo con lo que nos contaron los niños en las entrevistas de grupo el teléfono móvil sirve fundamentalmente a un propósito: mejorar el control de sus movimientos, lo que, paradójicamente, tiene un efecto liberador en muchos adultos al posibilitar un contacto distante constante.

G(13): Yo lo tengo desde los 10 años [teléfono móvil], pero porque me fui a una excursión con el colegio a Ávila y eran 12 horas de autobús y eso, porque nos teníamos que parar mucho. Y entonces mi madre me lo compró y no me lo iba a quitar cuando volviera. 
I: Lo compraron más que nada para que vuestros padres os pudieran llamar y saber si...

G(12): Para controlarnos.

G(12): Yo [lo utilizo] para mandar mensajes y, cuando salgo, llaman mis padres para saber dónde estoy. Y a mí me lo regalaron para eso. Porque si salgo y no saben dónde estoy, ellos me llaman y me preguntan dónde estoy.

$\mathrm{B}(11)$ :Yo es que lo utilizo para algo necesario, para salir con mis amigos, no. Por ejemplo, cuando vamos al cine, para poder llamar a nuestros padres.

$\mathrm{B}(13)$ : Ahora lo que están es más seguros, ¿̨no?, porque te pueden llamar y saber dónde estás.

Naturalmente, como ya hemos indicado, los niños aprenden pronto que este tipo de tecnología posibilita tanto el control como la ubicuidad, en la medida que la transgresión de la norma adulta vuelve del revés el uso del aparato y contribuye a proporcionar mayor autonomía a los menores. De esta manera puede burlarse la vigilancia de los adultos y obtener una especie de «independencia en la sombra» que dura lo que dura la ficción del control. A esto hay que unir que el trabajo directo con menores ha revelado que éstos, lejos de ser usuarios pasivos, ponen en marcha sus propios sistemas de gestión del riesgo (Olesen 1999).

I: ¿Y, desde que tenéis teléfono móvil, habéis notado que vuestros padres os dejan salir más tiempo?

G(13): Sí, pero ellos te preguntan dónde estás y tú les dices un lugar y estás en otro.

Poco a poco, conforme se profundiza en la información proporcionada por los menores, da la impresión de que el afán por proteger a los niños ha acabado enclaustrándolos en una rígida cuadrícula que ni tan siquiera ha sido diseñada conforme a sus intereses sino a los de sus progenitores y otras figuras adultas. Como afirma Dencik (1992), los niños del estado del bienestar viven sus vidas segregadas tras puertas que no pueden pillar los dedos y ventanas con cerraduras a prueba de niños. La consecuencia podría ser la del incremento en el control social de los menores y una mayor restricción en sus movimientos y su competencia social (Corsaro 1997; James y James 2001).

Los niños han recurrido con frecuencia a esta idea a la hora de expresarse libremente en el marco de los grupos realizados, donde han recalcado una y otra vez sentirse encorsetados en un papel excesivamente estrecho y saturado de implicaciones académicas. Encontramos, en uno de los grupos de preadolescentes y ambiente urbano, una niña con origen social medio-alto que expresó la idea con suficiente locuacidad:

G(13): También es que mi madre no puede pretender que yo me pase todo el día estudiando, tengo que tener un poco de libertad. Y si lo que me gusta a 
mí es hablar a mis amigos, ya que no puedo hablar a diario porque no me dejan salir de mi casa, ¿qué más le da que esté una hora conectada a Internet hablando con ellos?

De la misma manera, fue una constante durante el desarrollo de los grupos que el investigador aportara preguntas que iban explícitamente dirigidas a abordar este tema. Las más de las veces se interrogaba al grupo sobre sus preferencias, dándole a escoger entre una actividad de tipo sedentario (conectarse a Internet, jugar a videojuegos, etc.) y otra a efectuar en la calle (jugar al fútbol, pasear, etc.). La respuesta fue, casi invariablemente, que se prefería el juego activo en la calle antes que el ocio tecnológico, pero que éste no podía tener lugar por muy diferentes motivos. Como en el caso del discurso de la niña que hemos presentado antes, la tecnología se emplea muchas veces como una prótesis eficaz para producir contacto social cuando las condiciones no lo facilitaban o para llenar ratos de ocio que no son susceptibles de transcurrir en la calle.

I: Y yo os pregunto una cosa: si os gusta más jugar en la calle que estar jugando al ordenador, ¿por qué echáis tanto tiempo jugando al ordenador?

$\mathrm{B}(10)$ : Porque no llaman para jugar al fútbol.

B(11): Ahí está, aunque yo lo preferiría.

I: Parece que os gusta más estar con vuestros amigos que estar solos jugando a la Play...

$\mathrm{B}(11)$ : Es que siempre no puedes estar con tus amigos. Cuando es siesta o es muy tarde no puedes estar con ellos.

$\mathrm{B}(12)$ : $\mathrm{O}$ si se han ido tus amigos a algún sitio y estás solo, pues juegas en casa.

I: ¿Por qué no salís más con los amigos en lugar de estar jugando?

$\mathrm{B}(13)$ : Igual entre semana no se puede salir. $\mathrm{Y}$ además no puedes estar todo el día en la calle.

B(13): Por ejemplo, un fin de semana a las 12 de la noche pues te puedes poner a jugar un rato, ¿no? Yo no puedo salir a las 12.

Una de las evidencias que nos condujeron a la idea de que el supuesto aislamiento provocado por las TIC podría tratarse, en realidad, de una forma encubierta de sociabilidad, la constituye el análisis de la descripción infantil del uso de estas tecnologías, pero muy especialmente de las formas de contacto virtual que facilita la red de redes a través de aplicaciones como el chat o la mensajería instantánea. Internet se ha convertido en un lugar privilegiado para este contacto virtual, máxime si tenemos en cuenta que es difícilmente controlable por los padres y tutores. Así, la potencialidad de un medio que propicia encuentros de todo tipo y que presenta barreras importantes al control externo lo ha convertido en una aplicación muy popular entre los menores, 
que casi lo han convertido en un espacio propio dotándolo de sus propias reglas y fronteras. Internet es, ante todo, un lugar de encuentro para un grupo de sujetos que desbordan el reducido hábitat de la infancia que construyen los adultos. Son especialmente significativas las palabras que los chicos y chicas dedican al chat:

B (12): Es [el chat] donde se busca la gente para tener novio, para cosas así. $\mathrm{B}(12)$ : Chatear es una especie de sitio donde buscar novio, hacer nuevos amigos de otros sitios, hablar con gente, con amigos que tú conozcas.

B(11): Para divertirse..., mentir.

I: ¿Y qué hacéis para divertiros en Internet, a parte de lo del chat?

$\mathrm{B}(11)$ : Yo es que nada más que hago eso, entro en el ordenador, me conecto a Internet, abro el Messenger y me paso el tiempo que sea hablando con mis amigas.

En ocasiones, el instrumento que facilita la sociabilidad (la red, el chat) se convierte así mismo en producto consumido en el seno de esas mismas relaciones de proximidad, lo que da lugar a que se combinen dos redes diferentes de sociabilidad: una virtual, que tiene lugar en un entorno telemático; la otra física y real, convirtiendo el ordenador personal en un objeto de ocio compartido. La conducta de los niños en los conocidos popularmente como cíbers, donde pueden acceder libremente a Internet fuera del entorno doméstico, se ajusta a esta descripción. Normalmente el grupo acaba construyendo un uso grupal del chat:

G(12): En el cíber, cuando nos vamos al cine vamos un grupo de gente y, a lo mejor, nos ponemos a gastar bromas también con más gente.

I: Entonces, ¿̇ú quedas con tus amigos para entrar en Internet?

G(13): No, eso lo suelo hacer más los viernes y los sábados, en el cíber.

El caso del chat y otras formas de comunicación virtual como la mensajería instantánea ilustra de manera muy rica el tipo de apropiación grupal de la tecnología que generan los niños y preadolescentes. De hecho, son productos muy populares entre la población infantil por las razones antes apuntadas, al favorecer el contacto en circunstancias en que, de otra manera, sería imposible, y también por constituir un medio de interacción que no suele gozar de la fiscalización estricta por parte de los adultos. De hecho, la interacción del niño con estos nuevos medios resulta compleja y variable. En lo que respecta al contacto virtual, por ejemplo, encontramos en el discurso infantil frecuentes referencias a dos maneras de enfocar el uso del chat y la mensajería: (a) una basada en el contacto efímero con desconocidos, que tiende a producir relaciones frágiles aunque duraderas; la otra $(b)$ sustentada sobre la extensión de las redes sociales ya conocidas y prácticas en el entorno escolar del chico. 
Respecto al primer tipo de contacto, los niños no permanecen fuera de la tendencia a establecer relaciones efímeras, muy común también entre adultos, en las que habitualmente se oculta o manipula la identidad. Suelen producir vínculos frágiles, reconocibles en ciertos fragmentos del discurso infantil como el que reproducimos a continuación:

I: Entonces, cuando entras en un chat, ¿'hablas con gente que conoces?, ¿gente del colegio?

B(11): No, del colegio no, sobre todo hablo con una chiquilla que se llama... Tormenta.

I: ¿La has conocido en Internet?

B(11): Sí, claro.

I: ¿Sabes de dónde es?

B(11): Sí, es de Murcia, me parece que me dijo.

Además, son los propios niños los que informan sobre la relativa estabilidad y superficialidad de este tipo de contactos:

I: ¿Qué tal son las relaciones con esos amigos? ¿Os gustan?

$\mathrm{B}(13)$ : Es que ese tipo de relaciones es muy moderno, porque como no los ves y eso, tu siempre que cuando tienes mucha confianza con una persona y estás acostumbrada a verla siempre se hace un choque y discutes por algo, ¿no? Pero una relación que hablas, como mucho una hora con él, no le puedes...

Como ya se ha señalado, en ocasiones los propios niños afirman que mienten deliberadamente para producir una impresión favorable en su interlocutor: normalmente proporcionando información falsa sobre su edad. ${ }^{12} \mathrm{Sin}$ embargo, a tenor de la información encontrada en las entrevistas grupales, parece más frecuente el segundo tipo de sociabilidad expandida por medios virtuales: la que se basa en continuar en la red contactos preexistentes, normalmente amistades que provienen del entorno educativo del niño. Por supuesto, esto implica una fuerte complicidad entre los interlocutores, sobre todo a la hora de reconocerse, lo que se logra a través del apodo, alias o, vulgarmente, nickname:

I: Tú, cuando coges un alias, les dices a tus amigos cuál es tu alias, para que te reconozcan, ¿no?

B(11): Claro, y ellos también a mí.

12. Un niño de 11 años que participó en uno de los grupos de preadolescentes urbanos de clase media nos confesó, por ejemplo, que habitualmente informaba a sus contactos de que tenía 20 años y era una chica para, según sus propias palabras, «reírse de ellos». 
I: Entonces, a lo mejor, si chateáis os encontráis con amigos, ¿̨no?

$\mathrm{B}(11)$ : Sí, yo siempre me pongo mi alias para comunicarme.

$B(12)$ : Nunca ponemos nuestro nombre.

Y a partir de esta mutua encriptación de identidades y contando con la relativa seguridad que proporciona acudir a una lista de contactos en lugar de a un espacio de interacción completamente abierto, se produce la comunicación, preferentemente a través de algún sistema de mensajería instantánea:

B(13): En el chat tienes un montón de personas y tú vas hablando con quien quieres y no los conoces. Pero a lo mejor, en el Messenger ${ }^{13}$, tú tienes los que conoces.

I: Vosotros, entonces, normalmente, con el Messenger o con el chat, ¿qué es lo que hacéis?

G(11): Pues hablar con las personas que tú quieras.

I: Pero, esas personas, ¿quiénes son?

G(13): Amigos.

B(13): Algún amigo de por la mañana en el colegio que tú no lo ves. Nosotros hablamos cuando, a lo mejor, está el día malo y no podemos salir.

G(13): Y por ejemplo salimos, ¿̨no?, y llegamos a casa a las 10, pues a partir de las 10 para adelante estamos un rato hablando por Internet porque no nos dejan salir más de la habitación.

Esta convergencia entre redes de sociabilidad vinculadas a la propia cultura infantil y la experiencia grupal de la infancia y redes artificiales que descansan sobre la potencialidad de las TIC para incrementar el contacto social distante y fugaz, es una de las constantes de la apropiación de la tecnología por parte de los menores y una de las claves que explican el impacto de las mismas sobre la vida social infantil. Y tiende a reproducirse en otros ámbitos en la medida en que los menores también dan un uso social a las consolas portátiles de videojuegos o al propio PC. Por tanto, la imagen que debe emanar del discurso infantil conduce necesariamente hacia una complejización ineludible de los diagnósticos del impacto de las TIC sobre la vida social de los menores. Entre otras cosas, porque el pretendido efecto de aislamiento producido por la tecnología puede no tener lugar, al tiempo que es posible observar la dinámica

13. Existen multitud de sistemas de mensajería instantánea, pero uno de los más populares es el sistema Messenger de Microsoft basado en listas de contactos con los que es posible comunicar en tiempo real e intercambiar archivos e imágenes si se dispone de webcam, al estilo de una videoconferencia sostenida entre un número restringido de participantes. Estaba especialmente difundido entre los niños que participaron en los grupos urbanos. 


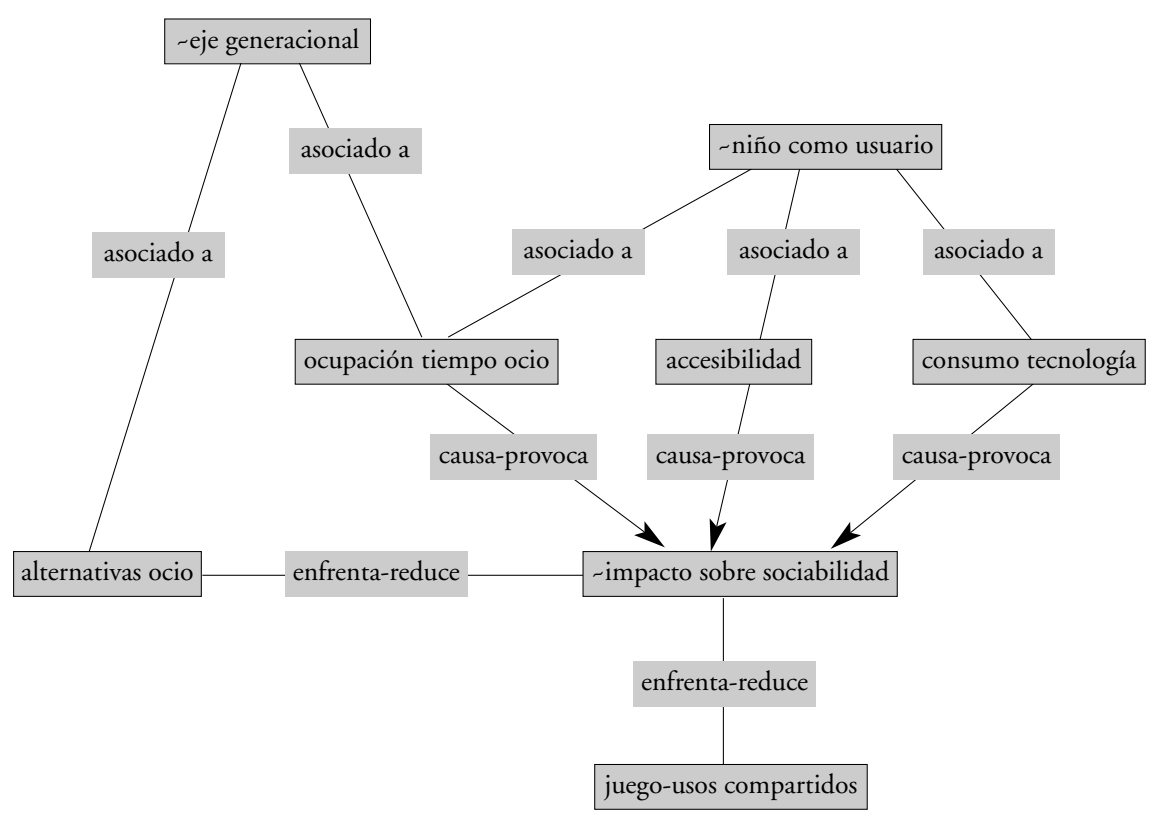

Figura 1. Impacto tecnológico sobre la sociabilidad infantil: red de fenómenos.

opuesta: la tecnología conduce hacia la proliferación del contacto social en un medio telemático.

En cualquier caso, esperamos haber ilustrado la red de fenómenos implicados en la apropiación de la tecnología por parte del menor, que representamos en la figura 1. En ella vemos como el impacto de la tecnología sobre la sociabilidad infantil es complejo e indeterminado, pues depende de otros factores tales como la destreza del menor como usuario, las alternativas de ocio disponibles en el marco de su vida cotidiana, así como la existencia de juegos y usos compartidos que vienen a reducir el peligro de atomización y aislamiento social. Junto a éstas, no parecen desdeñables las influencias debidas al control generacional ejercido por los adultos, la cuestión de la accesibilidad a los productos tecnológicos o la caracterización del niño como consumidor, en general, y como consumidor de tecnología en particular.

\section{A modo de conclusión}

A lo largo de este texto hemos tratado de transmitir fielmente las impresiones que produjo el trabajo directo con niños, en relación a la cuestión del impacto de las nuevas tecnologías de la información y la comunicación en sus vidas y más concretamente sobre sus redes sociales y sobre el contacto social con 
otros menores y con sus familias. Desde un comienzo, nos parecía que la literatura especializada sobre este tema producía una imagen distorsionada del menor, en tanto le restaba competencia y autonomía y lo convertía en un agente social «mutilado». Desde la sociología de la infancia se ha señalado repetidamente que el concepto o representación social de la infancia y la vida infantil se encuentra en la raíz de esta manera de entender la naturaleza de la vida de los niños. Queríamos comprobar si la realidad coincidía con el diagnóstico de la misma que esta literatura propaga, para lo cual tomamos contacto con niños de entre 6 y 13 años a través de un diseño cualitativo de investigación. El grueso de este trabajo lo ha constituido el análisis del discurso infantil que emergió durante el desarrollo de la investigación.

Precisamente, los principales hallazgos derivados del estudio del discurso infantil nos conducen a la impresión generalizada de que el niño que aparece representado en la literatura científica no constituye una aproximación precisa a la población infantil ni a sus intereses o quehaceres cotidianos, en la medida en que no refleja la complejidad de la apropiación grupal de los productos tecnológicos, característica de la infancia. En este sentido, a través de las entrevistas de grupo pudimos constatar que los niños entrevistados eran usuarios que poseían cierto grado de cualificación y dominio del medio tecnológico, a la vez que transgresores conscientes de las normas que los adultos —más concretamente sus tutores - imponen al mismo.

Así mismo, fueron hallazgos plausibles en el contexto del análisis de este discurso infantil la constatación de que:

a) La mayor parte de los niños, desde edades muy tempranas, eran buenos conocedores de las últimas aplicaciones tecnológicas y sus funciones, sin que las figuras parentales jueguen necesariamente un papel determinante en su uso ni en la cualificación que precede a su uso.

b) Su nivel de destreza, aún siendo alto si lo comparamos con la representación del niño que encontramos en la literatura científica, no es uniforme y tiende a crecer con la edad (más alto en preadolescentes) así como con la extracción social y el hábitat (más alto en niños y niñas de contexto urbano y clase social media-alta).

c) La penetración tecnológica en los hogares no puede explicarse sino acudiendo a una compleja interacción entre las necesidades de los adultos y de los propios niños. Así, hemos encontrado aplicaciones tecnológicas que, si bien son de uso común entre los menores consultados, tienen su origen en la necesidad parental de incrementar el control sobre los desplazamientos de los niños (telefonía móvil). El papel de los adultos en la relación que los niños establecen con las nuevas tecnologías de la información y la comunicación, por tanto, debe ser replanteado.

d) Respecto a la cuestión del supuesto aislamiento producido por estas nuevas tecnologías, y muy especialmente por la irrupción de Internet en la vida cotidiana de los menores, el discurso infantil parece conducir a una importante matización: es posible que el aislamiento sea un fenómeno preexis- 
tente a estas tecnologías y haya facilitado su proliferación. A tenor de lo que expresaron los sujetos en las entrevistas de grupo, la mayor parte de ellos dan un uso social a un equipamiento concebido para ser usado individualmente. A esto hay que añadir que uno de los principales usos de la red es, precisamente, el de producir contactos virtuales en condiciones en los que el contacto cara a cara no es posible.

e) Por tanto, podríamos no sólo rechazar la denominación «aislamiento» para referirnos a la proliferación de estos contactos virtuales, que pueden ser con desconocidos o (más frecuentemente) con la red próxima del niño, sino sustituirla por la de «sociabilidad expandida» al referirnos a que la tecnología constituye una suerte de medio a través del cual el niño incrementa su contacto social en condiciones en las que existen barreras para que ésta tenga lugar en el mundo real y entornos no-telemáticos. De hecho, la retirada del niño del espacio urbano, y más concretamente de la calle como espacio de juego, puede ser uno de los fenómenos subyacentes a todo este proceso de penetración tecnológica en la vida social infantil.

Por tanto, más estudios empíricos de esta naturaleza, que tratan de superar los enfoques deterministas, reduccionistas y/o esencialistas en torno a la cuestión del impacto de las nuevas tecnologías sobre la vida del niño, son necesarios para corregir la divergencia existente entre la representación social del niño presente en la literatura científica, que aboca a la descripción de un agente social inmaduro, vulnerable y descualificado, y la realidad tal y como se presenta cuando contamos con el propio niño como informante en un contexto no-experimental. En definitiva, los modelos reduccionistas afines al llamado human computer interaction difícilmente pueden captar la riqueza de matices presente en la realidad social de la infancia, ni el desarrollo creciente de la autonomía del niño en tanto agente social y usuario de productos tecnológicos, y esto puede derivar en una percepción distorsionada de la naturaleza del fenómeno de la innovación tecnológica y su penetración en la vida cotidiana de los niños.

\section{Bibliografía}

AIMC (2004). «Audiencia infaltil/juvenil de Medios». [Acceso on-line en: www.aimc.es] Bericat Alastuei, Eduardo (1996). "La sociedad de la información: tecnología, cultura, sociedad». Revista Española de Investigaciones Sociológicas 76: 99-121. Brannen, Julia; O’Brien, Margaret (1996). Children in Families: Research and Policy. Londres: Falmer Press.

CASTELLS, Manuel (1995). La ciudad informacional. Tecnologías de la información, reestructuración económica y el proceso urbano-regional. Madrid: Alianza.

- (1997). La era de la información. Economía, sociedad y cultura. Vol. I: La sociedad red. Madrid: Alianza.

CORSARO, William A. (1992). «Interpretative Reproduction in Children's Peer Cultures». Social Psychology Quarterly 55: 160-177.

- (1997). The Sociology of Childhood. Thousand Oaks (Calif.): Pine Forge Press. 
Corsaro, William A.; Rizzo, Thomas A. (1990). «An Interpretative Approach to Childhood Socialization». American Sociological Review 53: 879-894.

DENCIK, Lars (1992). "Creciendo en la era posmoderna: el niño y la familia en el Estado del Bienestar». Anuario de Psicología 53: 71-98.

FAGER, Keri et al. (2001). "Constructing the Child Computer Use: from Public Policy to Private Practices». British Journal of Sociology of Education 22 (1): 91-108.

Fine, Gary A.; SANDSTROM, Kent L. (1988). Knowing Children. Participant Observation with Minors. Newbury Park (Calif.): Sage Publications.

FreEMAN-Longo, Robert E. (2000). «Children, Teens and Sex on the Internet». Sexual Addiction \& Compulsivity 7 (1-2): 75-90.

Graue, Elizabeth; Walsh, Daniel J. (1998). Studying Children in Context. Theories, Methods, and Ethics. Thousand Oaks (Calif.): Pine Forge Press.

GreIG, Anne; TAYLOR, Jayne (1999). Doing Research with Children. Londres: Sage.

HicK, Steven; HalPIN, Edward (2001). "Children's Rights and the Internet». Annals of the American Academy of Political and Social Sciences 575: 56-70.

Holmes, Robyn M. (1998). Fieldwork with Children. Thousand Oaks (Calif.): Pine Forge Press.

Holloway, Sarah L.; Valentine, Gill (2001). "“It’s Only as Stupid as You Are”: Children and Adult's Negociation of ICT Competence at Home and at School». Social and Cultural Geography 2 (1): 25-42.

HonIG, Michael-Sebastian et al. (Hrsg.) (1999). Aus der Perspektive von Kindern? Zur Methodologie der Kindheitsforschung. Munich: Juventa-Verlag.

INE (2001). Estadisticas de la sociedad de la información. Madrid: Instituto Nacional de Estadística.

- (2003). Encuesta de tecnologías de la información en los hogares 2003. Madrid: Instituto Nacional de Estadística. [Acceso on-line en: www.ine.es]

JACKSON, Phillip W. (1991). La vida en las aulas. Madrid: Morata.

JAMES, Adrian L.; JAMES, Allison (2001). «Tightening the Net: Children, Community, and Control». British Journal of Sociology 52 (2): 211-228.

JameS, Allison; Prout, Alan (ed.) (1997). Constructing and Reconstructing Childhood: Contemporary Issues in the Sociological Study of Childhood. Londres: Falmer Press.

Jenks, Chris (ed.) (1982). The Sociology of Childhood. Esential Readings. Londres: Greggs Revivals.

JENKS, Chris (1996). Childhood. Londres: Routledge.

Jessen, Carsten (1999). Children's Computer Culture. Three Essays on Children and Computers. Odense: Department of Contemporary Cultural Studies (University of South Denmark).

JuÁreZ, Miguel (1998). "Cambios sociales que afectan al menor y la familia». En RODRíGUEZ TORRENTE, Jesús (ed.). El menor y la familia. Madrid: Universidad Pontificia de Comillas.

Kofler, A. (1998). «Digital Europe 1998: Policies, Technological Development and Implementation of the Emerging Information Society». Innovation 11 (1).

MCCABE, Kimberly A. (2000). "Child Pornography and the Internet». Social Science Computer Review 18 (1): 73-76.

Moll, Marita (1998). «No More Teachers No More Schools: Information Technology and the Deschooled Society». Technology in Society 20: 357-369.

MONTGOMERY, Kathryn C. (2000). "Children's Media Culture in the New Millenium: Mapping the Digital Landscape». The Future of Children. Children and Computer Technology 10 (2): 145-167. 
NisSENBAum, Helen; WaLKer, Derek (1998). «Will Computers Dehumanize Education? A Grounded Approach to Values at Risk». Technology in Society 20: 237-273.

Olesen, Jesper (1999). Children and Media Risks. Odense: Department of Contemporary Cultural Studies (University of South Denmark).

OrLeAns, Myron; LANEY, Margaret C. (2000). "Children's Computer Use in Home: Isolation or Sociation». Social Science Computer Review 18 (1): 56-72.

Oswell, David (1998). "The Place of "Childhood" in Internet Content Regulation". International Journal of Cultural Studies 1 (2): 271-291.

Postman, Neil (1984). The Dissappeareance of Childhood. Nueva York: Delacorte Press.

QVORTRUP, Jens (1987) (ed.). «Sociology of Childhood: Introduction». International Journal of Sociology 17 (3): 3-37.

Qvortrup, Jens (1993). "Nine Theses About "Childhood as a Social Phenomenon"». En QvorTruP, Jens (ed.). Childhood as a Social Phenomenon. Lessons from an International Project. Viena: European Centre for Social Welfare Policy and Research.

Qvortrup, Jens et al. (1994). Childhood Matters: Social Theory, Practice and PoliTIC. Viena: Avebury.

STANLEY, Janet (2001). "Child Abuse and the Internet». Issues in Child Abuse Prevention 15. [Acceso on-line en: www.aifs.org.au el 15/2/2002]

Subrahmanyam, Kaveri et al. (2000). «The Impact of Home Computer Use on Children's Activities and Development». The Future of Children. Children and Computer Technology 10 (2): 123-144.

TIEN, Lee (1994): "Children's Sexuality and the New Information Technology: A Foucaultian Approach». Social and Legal Studies 3: 121-147.

Wartella, Ellen A.; Jennings, Nancy (2000). «Children and Computers: New Technology-Old Concerns». The Future of Children. Children and Computer Technology 10 (2): 31-43.

Wright Mills, C. (1961). La imaginación sociológica. México: Fondo de Cultura Económica.

Yates, Simeon J.; Littleton, Karen (1999). «Understanding Computer Game Culture». Information, Communication and Society 2 (4): 566-583.

Zelizer, Viviana (1985). Pricing the Priceless Child: The Changing Social Value of Children. Nueva York: Basic Books. 